\title{
Spin-liquid model of the sharp resistivity drop in $\mathrm{La}_{1.85} \mathrm{Ba} a_{0.125} \mathrm{CuO}$.
}

\author{
A. V. Chubukov ${ }^{1}$ and A. M. Tsvelik ${ }^{2}$ \\ 1 Department of University of Wisconsin, Madison, WI 53706, USA and \\ 2 Department of Condensed Matter Physics and Materials Science, \\ Brookhaven National Laboratory, Upton, NY 11973-5000, USA
}

(Dated: November 24, 2018)

\begin{abstract}
We use the phenomenological model proposed in our previous paper [Phys. Rev. Lett. 98, 237001 (2007)] to analyse the magnetic field dependence of the onset temperature for two-dimensional fluctuating superconductivity $T^{* *}(H)$. We demonstrate that the slope of $T^{* *}(H)$ progressively goes down as $H$ increases, such that the upper critical field progressively increases as $T$ decreases. The quantitative agreement with the recent measurements of $T^{* *}(H)$ in $L a_{1.85} \mathrm{Ba}_{0.125} \mathrm{CuO}_{4}$ is achieved for the same parameter value as was derived in our previous publication from the analysis of the electron self energy.
\end{abstract}

Recent experiments on $\mathrm{La}_{1-x} \mathrm{Ba}_{x} \mathrm{CuO} \mathrm{O}_{4}$ at $x=1 / 8$ [1] revealed a complex hierarchy of energy scales in this material. It displays a charge ordering transition at $T_{c o}=54 \mathrm{~K}$, a spin ordering transition at $T_{\text {spin }}=42 \mathrm{~K}$ with a subsequent one order of magnitude drop in the in-plane resistivity, the Berezinskii-Kosterlitz-Thouless (BKT) transition to a two-dimensional superconductivity at $T_{B K T}=16 K$, a crossover from $2 \mathrm{D}$ to $3 \mathrm{D}$ regime around $10 K$, and a transition to a true $3 \mathrm{D}$ superconductivity at $4 K$. This hierarchy is summarized and discussed in detail in 2].

It turns out that the temperature $T^{* *}$ where the resistivity crossover occurs is sensitive to the $c$-axis magnetic field which separates this phenomenon separately from the spin ordering. In this paper, we address the issue of this crossover. The measurements performed in a magnetic field [1] revealed that (i) $T^{* *}$ marks the onset of fluctuational diamagnetism, and (ii) $T^{* *}$ decreases with the field. These two effects and the fact that the resistivity sharply drops $T^{* *}$ are consistent with the idea that $T^{* *}$ marks the onset of a fluctuational pairing regime without (quasi-) long-range superconducting order. The details of the system behavior near $T^{* *}$, however, depend on the underlying model. The authors of [2] considered a model of weakly coupled parallel superconducting stripes. Within this model, $T^{* *}$ is the temperature at which the inter-stripe coupling becomes strong, and a vortex liquid is formed.

We propose another explanation, based on the model with a flat Fermi surface in the antinodal regions near $(0, \pi)$ and $(\pi, 0)$ points in the Brillouin zone 3]. Fermions in these regions form two quasi-1D spin liquids coupled by Josephson-type interaction. In this model, the pairing amplitudes in the antinodal regions are developed at $T^{*} \gg T^{* *}$ due to the attractive interactions in the spinliquid state, however, phase fluctuations at $T>>T^{* *}$ are effectively one-dimensional, and are pinned by the defects. At $T^{* *}$, the Josephson coupling becomes sufficiently strong to lock the relative phase of the two order parameters at $\pi$, and the system response becomes two-dimensional. This leads to depinning of the phase

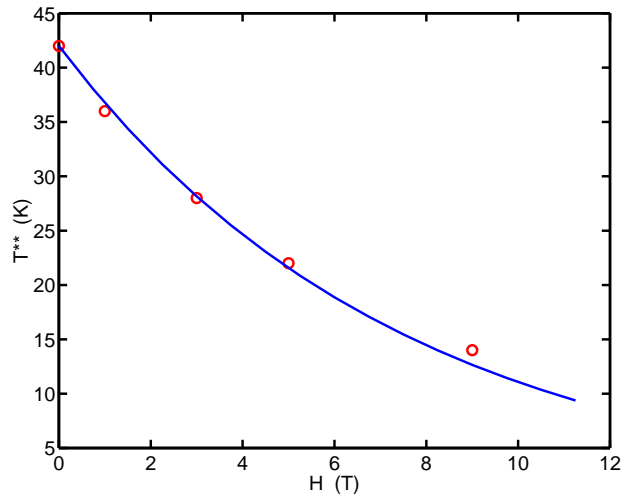

FIG. 1: The plot of $T^{* *}(H)$. The points are the data from Ref. [1], the solid curve is the exponential fit by Eq. (1).

fluctuations resulting in the drop in the resistivity. Still, because of vortices in the $2 \mathrm{D}$ regime, the (quasi)-longrange superconducting order develops only at a smaller $T_{c}<T^{* *}$.

Just like the model of parallel stripes [2], our model of "crossed stripes" near $(0, \pi)$ and $(\pi, 0)$ explains qualitatively the resistivity drop, the absence of fluctuational diamagnetism above $T^{* *}$, and the sensitivity of $T^{* *}$ to a magnetic field. [5]. However, the measurements of $T^{* *}(H)$ put an additional constraint on the theory - not only $T^{* *}$ decreases with the field, but $\left|d T^{* *} / d H\right|$ also $d e$ creases as $H$ goes up, i.e., at very low $T$, the critical field below which the system response is two-dimensional, becomes very large. The data for $H<9 T$ can be well fitted by the exponential dependence (see Fig. 1):

$$
\frac{T^{* *}(H)}{T^{* *}(0)}=\exp \left(-H / H_{0}\right), \quad H_{0} \approx 7.5 T
$$

For such $T^{* *}(H),\left|d T^{* *} / d H\right|$ exponentially decreases as $H$ increases. If this trend continued to higher $H$, the critical field $H_{c 2}(T)$ defined as $T^{* *}\left(H_{c 2}\right)=T$ would become infinite at $T=0$.

The $H$ dependence of $T^{* *}$ for Josephson-coupled stripes running parallel to each other in the $2 \mathrm{D}$ plane, 
i.e., for the same model as in Ref. [2] was considered by Carr and one of us [4]. It was found that the slope of $d T^{* *} / d H$ increases with decreasing $T$, and $H_{c 2}$ remains finite at $T=0$, in qualitative disagreement with the data. We demonstrate below that our model of crossed stripes located near $(0, \pi)$ and $(\pi, 0)$ yields the behavior of $T^{* *}(H)$ in a good agreement with the measurements. Thus we show that the slope of $d T^{* *} / d H$ decreases with increasing $H$ for any value of the scaling dimension $d$ of the superconducting order parameter. To achive a quantitative agreement with the experimental fit (1) we have to set $d \approx 1 / 2$. We have to remind the reader that in $[3]$ the same value of $d$ was postulated on the basis of analysis of the electron self energy. This gives an important check for self-consistency of the theory.

We associate $T^{* *}(H)$ with the instability of a $2 \mathrm{D}$ pairing susceptibility in the random phase approximation (RPA). Fluctuations beyond RPA transform the instability into a crossover [3]. In zero field, the RPA expression for the susceptibility reads, in momentum space

$$
\chi\left(k_{x}, k_{y}\right)=\chi_{0}\left(k_{x}\right)+J^{2} \chi\left(k_{x}, k_{y}\right) \chi_{0}\left(k_{x}\right) \chi_{0}\left(k_{y}\right)
$$

where $\chi_{0}(k)$ is the $1 \mathrm{D}$ static pairing susceptibility [6]:

$$
\chi_{0}(k)=\frac{2}{\Delta^{2}}\left[\sin \pi d \Gamma^{2}(1-d)\left(\frac{2 \pi T}{\Delta}\right)^{-2+2 d}\left|\frac{\Gamma(d / 2+i v q / 4 \pi T)}{\Gamma(1-d / 2+i v q / 4 \pi T)}\right|^{2}-\frac{\pi}{1-d}\right]
$$

Here $\Gamma(\ldots)$ are $\Gamma$-functions, $d<1$ is the scaling dimension of the superconducting order parameter, $v$ is the velocity of the phase mode, and $\Delta$ is the ultraviolet cutoff. The last term in $\chi_{0}$ can be neglected as we will only consider $T \ll \Delta$, when the first term in (3) dominates. Parameters $v$ and $d$ are free parameters of our theory and should be extracted from the experiments in the $T$ region where the superconducting phase fluctuations are essentially one-dimensional (that is, at $T$ below the spin gap, but larger than $T^{* *}$ ). In [3] we found that the best agreement with the photoemission experiments is obtained when $d \approx 1 / 2$. As we will see, this value is also favored by the observed $T^{* *}(H)$ dependence.

Taking a Fourier transform over $k_{x}$, but leaving $k_{y}$ intact, we obtain from (2):

$$
\begin{aligned}
& \chi_{k_{y}}\left(x-x_{1}\right)= \\
& \chi_{0}\left(x-x_{1}\right)+J^{2} \int d x^{\prime} \chi_{0}\left(k_{y}\right) \chi_{0}\left(x-x^{\prime}\right) \chi_{k_{y}}\left(x^{\prime}-x_{1}\right)
\end{aligned}
$$

In a magnetic field, $k_{y} \rightarrow k_{y}+H x^{\prime}$ (we set $2 e / c=1$ ). Setting $k_{y}=0$ and $x_{1}=0$, we obtain integral equation for $\chi(x)=\chi_{k_{y}=0}(x)$ in the form

$$
\chi(x)=\chi_{0}(x)+J^{2} \int d x^{\prime} \chi_{0}\left(x-x^{\prime}\right) \chi\left(x^{\prime}\right) \chi_{0}\left(H x^{\prime}\right)
$$

where $\chi_{0}\left(H x^{\prime}\right)$ is given by (3) for $k=H x^{\prime}$, and $\chi_{0}(x)$ is the Fourier transform of $\chi_{0}(k)$. The temperature $T^{* *}(H)$ is the one at which $\chi(x)$ diverges.

Weak fields. Consider first the case when the magnetic field is weak, i.e., $T^{* *}(H)=T^{* *}(0)(1-\delta T)$, and $\delta T \ll 1$. A simple analysis shows that the parametrical condition for a weak field is $v^{2} H / T<<1$. Expanding
$\chi_{0}\left(H x^{\prime}\right)$ in $H$, we obtain from (3)

$$
\chi_{0}\left(H x^{\prime}\right)=B_{d}\left(\frac{2 \pi T}{\Delta}\right)^{2 d-2}\left[1-A_{d}\left(\frac{v H x^{\prime}}{\pi T}\right)^{2}\right]
$$

where

$$
\begin{aligned}
& A_{d}=\frac{1}{16}\left[\psi^{(1)}(d / 2)-\psi^{(1)}(1-d / 2)\right] \\
& B_{d}=\frac{2}{\Delta^{2}} \sin \pi d \Gamma^{2}(1-d) \frac{\Gamma^{2}(d / 2)}{\Gamma^{2}(1-d / 2)}
\end{aligned}
$$

and $\psi^{(1)}(x)$ is the derivative of the diGamma function.

Substituting (6) into (5), we obtain an integral equation for $\chi(x)$ in the form

$$
\begin{aligned}
& \chi(x)=\chi_{0}(x)+J^{2} \int d x^{\prime} \chi_{0}\left(x-x^{\prime}\right) \chi\left(x^{\prime}\right) \chi_{0}(0) \\
& -J^{2} \chi_{0}(0) A_{d} \frac{v^{2} H^{2}}{(\pi T)^{2}} \int d x^{\prime} \chi_{0}\left(x-x^{\prime}\right) \chi\left(x^{\prime}\right)\left(x^{\prime}\right)^{2}
\end{aligned}
$$

where $\chi_{0}(0)=\chi_{0}(k=0)$. Taking Fourier transform back to momentum space $\left(x \rightarrow k_{x}=k\right)$, and integrating by parts, we re-write the integral equation for $\chi$ as

$$
\begin{aligned}
& \chi(k)\left[1-J^{2} \chi_{0}(k) \chi_{0}(0)\right]-J^{2} \chi_{0}(k) \chi_{0}(0) \frac{A_{d} v^{2} H^{2}}{(\pi T)^{2}} \chi^{\prime \prime}(k) \\
& =\chi_{0}(k)
\end{aligned}
$$

This can be re-expressed as

$$
\left(\epsilon+c_{1} k^{2}-c_{2} \frac{\partial^{2}}{\partial k^{2}}\right) \chi(k)=\chi_{0}(k)
$$

where $\epsilon=1-\left(T^{* *}(0) / T\right)^{4-4 d}, \quad c_{1}=A_{d} v^{2} /(\pi T)^{2}$, $c_{2}=A_{d} v^{2} H^{2} /(\pi T)^{2}$, and we defined $T^{* *}(0)=$ 
$(\Delta / 2 \pi)\left(B_{d} J\right)^{1 /(2-2 d)}$. This agrees with the zero-field transition temperature in [3]. Expanding now in the eigenvalues of the differential equation as

$$
\chi(k)=\sum_{n} a_{n} \chi_{n}(k), \quad \chi_{0}(k)=\sum_{n} a_{n}^{(0)} \chi_{n}(k)
$$

where $\chi_{n}(k)$ are the solutions of

$$
\left(c_{1} k^{2}-c_{2} \frac{\partial^{2}}{\partial k^{2}}\right) \chi_{n}(k)=\epsilon_{n} \chi_{n}(k),
$$

we obtain

$$
a_{n}=\frac{a_{n}^{(0)}}{\epsilon+\epsilon_{n}}
$$

The eigenvalues of Eq. (12) can be easily obtained as (12) can be re-expressed as a harmonic oscillator

$$
-\frac{1}{2 M} \frac{\partial^{2} \chi_{n}(k)}{\partial k^{2}}+\frac{M \omega^{2} k^{2}}{2} \chi_{n}(k)=\epsilon_{n} \chi_{n}(k)
$$

where $\omega^{2}=4 c_{1} c_{2}$ and $M^{-1}=2 A_{d}(v / \pi T)^{2}$. The eigenfunctions of (14) are $\epsilon_{n}=\omega(n+1 / 2)$, the lowest one is $\epsilon_{0}=\omega / 2=A v^{2} H /(\pi T)^{2}$. From (13), the instability in the field occurs when $\epsilon+\epsilon_{0}=0$, i.e, when $T=T^{* *}(H)=T^{* *}(0)(1-\delta T)$, where

$$
\delta T \approx \frac{1}{4(1-d)} \frac{A_{d} v^{2} H}{\left(\pi T^{* *}(0)\right)^{2}}
$$

We see that at small fields, $T^{* *}(H)$ decreases linearly with $H$. The linear dependence at small fields is also present in the model of parallel stripes [4]. If we formally extrapolate the small-field result to $T=0$, we obtain the upper critical field

$$
H_{c 2}^{e x t r}(T=0)=\left(\frac{\Delta}{v}\right)^{2}\left(J B_{d}\right)^{1 /(1-d)} \frac{1-d}{A_{d}}
$$

The actual $H_{c 2}(T=0)$ is somewhat smaller in the model of parallel stripes [4], but, as we will see, is much larger than (16) in our model of crossed stripes.

Strong fields. Consider now the opposite limit of vanishing $T$, when $v^{2} H / T>>1$, i.e., the expansion in the field is no longer possible. In this limit, we have from (3)

$$
\chi_{0}\left(H x^{\prime}\right)=\frac{\bar{B}_{d}}{\left|H x^{\prime}\right|^{2-2 d}}
$$

where

$$
\begin{aligned}
& \bar{B}_{d}=(8 / \Delta)^{2} \sin (\pi d) \Gamma^{2}(1-d)\left(v^{2} / 4 \Delta^{2}\right)^{d} \\
& =B_{d}(2 \Delta / v)^{2-2 d}\left(\Gamma^{2}(1-d / 2) / \Gamma^{2}(d / 2)\right) .
\end{aligned}
$$

Instead of Eq. (9), we now have

$$
\chi(k)=\chi_{0}(k)\left[1+J^{2} \frac{\bar{B}_{2 d-1}}{H^{2-2 d}} \int d q \chi(q) \int d x^{\prime} \frac{e^{i(k-q) x^{\prime}}}{\left|x^{\prime}\right|^{2-2 d}}\right]
$$

Using

$$
\int d x^{\prime} \frac{e^{i(k-q) x^{\prime}}}{\left|x^{\prime}\right|^{2-2 d}}=\frac{\Gamma(2 d-1) \sin \pi d}{|k-q|^{2 d-1}}
$$

and introducing

$$
\chi(k)=\frac{\bar{B}_{d}}{|k|^{2-2 d}} \tilde{\chi}(k)
$$

and $d=(1+\epsilon) / 2$, we obtain from (19)

$$
\tilde{\chi}(k)=1+\frac{J^{2} \bar{B}_{d}^{2} \cos (\pi \epsilon / 2) \Gamma(\epsilon)}{H^{1-\epsilon}} \int d q \frac{\tilde{\chi}(q)}{|q|^{1-\epsilon}|k-q|^{\epsilon}}
$$

It is convenient to re-express this equation in the operator form, as $\hat{L} \tilde{\chi}(k)=1$, and expand in the eigenfunctions of the operator $\hat{L}$, which we label as $\tilde{\chi}_{m}(k)$. We get

$$
\tilde{\chi}(k)=\sum_{m} \frac{a_{m}^{(0)}}{1-\lambda_{m}} \tilde{\chi}_{m}(k)
$$

where $a_{m}^{(0)}$ are constants. The eigenvalues $\lambda_{m}$ are the solutions of

$$
\hat{L} \tilde{\chi}_{m}(k)=\left(1-\lambda_{m}\right) \tilde{\chi}_{m}(k)
$$

where

$$
\hat{L} \tilde{\chi}_{m}(k)=\tilde{\chi}_{m}(k)-\frac{J^{2} \bar{B}_{d}^{2} \cos \pi \epsilon / 2 \Gamma(\epsilon)}{H^{1-\epsilon}} \int d q \frac{\tilde{\chi}_{m}(q)}{|q|^{1-\epsilon}|k-q|^{\epsilon}}
$$

Eq. (25) was studied in the context of non-BCS superconductivity (with frequency instead of momentum) [7]. A similar equation has been studied in the content of superconductivity in graphene [8]. For $\epsilon>0$, the normalized solution of (25) with the largest eigenvalue is

$$
\tilde{\chi}_{m}(k)=\frac{1}{|k|^{\epsilon}}
$$

and the eigenvalue is

$$
\lambda_{0}=\frac{J^{2} \bar{B}_{d}^{2}}{H^{1-\epsilon}} \Psi_{\epsilon}, \quad \Psi_{\epsilon}=\frac{\pi^{2}}{2} \frac{1}{\Gamma^{2}(1-\epsilon / 2)(\sin \pi \epsilon / 4)^{2}}
$$

The critical field $H_{c 2}(T=0)$ is determined from $\lambda_{0}=1$ and is given by

$$
H_{c 2}(T=0)=\left[J^{2} \bar{B}_{d}^{2} \Psi_{\epsilon}\right]^{1 /(1-\epsilon)}
$$

In explicit form, we have 


$$
\begin{aligned}
& H_{c 2}(T=0)=\left(J \bar{B}_{d}\right)^{1 /(1-d)}\left(\frac{2 \Delta}{v}\right)^{2}\left(\frac{8}{(2 d-1)^{2}}\right)^{1 / 2(1-d)}\left[\frac{\Gamma(1-d / 2)}{\Gamma(d / 2)}\right]^{2 /(1-d)} \\
& =H_{c 2}^{e x t r}(T=0)\left[\left(\frac{4 A_{d}}{1-d}\right)\left(\frac{8}{(2 d-1)^{2}}\right)^{1 / 2(1-d)}\left[\frac{\Gamma(1-d / 2)}{\Gamma(d / 2)}\right]^{2 /(1-d)}\right]
\end{aligned}
$$

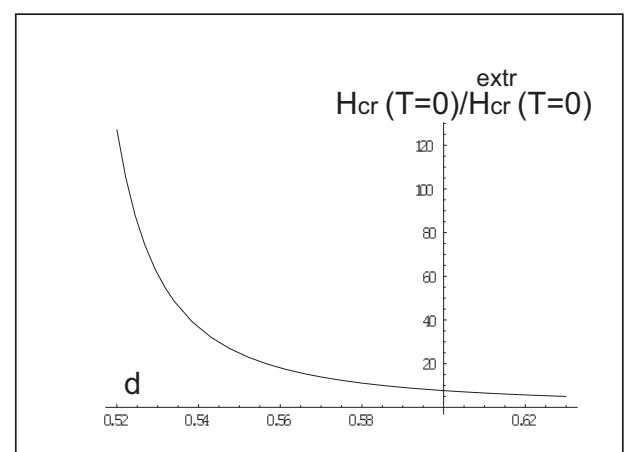

FIG. 2: The ratio $H_{c 2}(T=0) / H_{c 2}^{e x t r}(T=0)$ as a function of scaling dimension $d$, from Eq.(29). The ratio diverges logarithmicaly at $d \rightarrow 0.5$.

One can easily make sure that the actual $H_{c 2}(T=0)$ is much larger than $H_{c 2}^{e x t r}(T=0)$ for all $d \leq 1 / 2$ for which our computational scheme is applicable. Furthermore, as $d$ approaches $1 / 2, H_{c 2}(T=0)$ tends to infinity because $\Psi(\epsilon)$ diverges at vanishing $\epsilon=2 d-1$ as $\Psi_{\epsilon} \approx 8 / \epsilon^{2}$. The plot of the ratio $H_{c 2}(T=0) / H_{c 2}^{e x t r}(T=0)$ is presented in Fig. 2.

For $d \leq 1 / 2$, the analysis has to be modified to account for the divergence at $q=0$ in the r.h.s. of (25). The expected result is that $H_{c 2}$ becomes infinite at zero temperature. The divergence is power-law for $\epsilon<0$, and logarithmical at $\epsilon=0$. In the latter case,

$$
\chi_{0}\left(H x^{\prime}\right)=\frac{\bar{B}_{\epsilon=0}}{\left|H x^{\prime}\right|}
$$

and the RPA equation for $\chi(x)$ in the real space becomes

$\chi(x)=-2 \log T|x|-\frac{2\left(J \bar{B}_{\epsilon=0}\right)^{2}}{H} \int \frac{d x^{\prime}}{\left|x^{\prime}\right|} \chi\left(x^{\prime}\right) \log \left(T\left|x-x^{\prime}\right|\right)$

With the logarithmic accuracy, we can approximate

$$
\log \left(\left|x-x^{\prime}\right|\right) \approx \theta\left(x-x^{\prime}\right) \log |x|+\theta\left(x^{\prime}-x\right) \log \left|x^{\prime}\right|
$$

Substituting into (31), we re-write it as a differential equation

$$
\partial_{\zeta}^{2} \chi+\frac{4\left(J \bar{B}_{\epsilon=0}\right)^{2}}{H} \chi=-2 \partial_{\zeta}^{2} \log \left(T\left|e^{\zeta}-1\right|\right)
$$

where $\zeta=\log |x|$. The analysis of this equation shows that the susceptibility diverges at $H=H_{c 2}(T) \propto|\log T|$. This is equivalent to $T^{* *}(H) \propto \exp -H / H_{0}$, in agreement with Eq. (11). We see therefore that the high field dependence is well captured by our model with $d \approx 1 / 2-$ the same as we used in the previous work [3] to fit the normal state self-energy.

To summarize, we analyzed the behavior of $T^{* *}(H)$ (or, equivalently $H_{c 2}(T)$ ) in the model of two onedimensional spin liquids near $(0, \pi)$ and $(\pi, 0)$ coupled by Josephson-type interaction. For weak fields we found that $T^{* *}$ decreases linearly with $H$. Extrapolating this dependence down to zero temperature yields the extrapolated field $H_{c 2}^{e x t r}(T=0)$. Considering the strong fields we found that the actual $H_{c 2}(T=0)$ is always larger than the extrapolated value. The ratio $H_{c 2}(T=$ $0) / H_{c 2}^{e x t r}(T=0)$, characterizing the convexity of the $H_{c 2}(T)$-curve, increases when $d$ decreases and becomes infinite at $d \leq 1 / 2$. This convex behavior is consistent with the data, and has to be contrasted with the concave behavior for the model of parallel stripes. As a further evidence in support of our model, we found that the experimental $H_{c 2}(T)$ are well described by the theoretical formula with the scaling dimension of the $1 \mathrm{D}$ superconducting order parameter $d \approx 1 / 2$. The same $d$ provides the best fit to the photoemission data, as we argued earlier [3]. We think that all these give our model a considerable advantage in treating $L a_{1.85} \mathrm{Ba}_{0.125} \mathrm{CuO}$.

We acknowledge useful discussions with E. Fradkin, S. Kivelson, D. Scalapino and J. Tranquada and to J. Tranquada for kindly providing us Fig. 1. The research was supported by nsf-dmr 0604406 (A. V. Ch.), and by US-DOE under contact number DE-AC02-98 CH 10886 (A.M.T.). AVC acknowleges the support from the Theory Institute for Strongly Correlated and Complex Systems at BNL.

[1] Q. Li, M. Hucker, A.M. Tsvelik, and J.M. Tranquada, cond-mat/070337. 
[2] E. Berg, E. Fradkin, E-A Kim, S.A. Kivelson, V. Oganesyan, J. Tranquada, and S. Zhang, cond-mat/0704124).

[3] A.M. Tsvelik and A.V. Chubukov, Phys. Rev. Lett. 98, 237001 (2007).

[4] S. T. Carr and A.M. Tsvelik, Phys. Rev. B65, 195121 (2002).

[5] The underlying physics behind our model is quite different from the one in the standard model of interacting stripes. From mathematical perspective, however, our model does indeed look like a model of intersecting 1D stripes, coupled by Josephson-like interaction.

[6] H.J. Schulz and C. Bourbonnais, Phys. Rev. B27, 5856 (1983).

[7] A. Abanov, A.V. Chubukov, and A.M. Finkelstein, Europhys. Lett., 54, 488 (201); A. Abanov, B.L. Altshuler, A.V. Chubukov, and E. Yuzbashyan, unpublished.

[8] D.V. Khveshchenko and W.F. Shively, Phys. Rev. 73, 115104 (2006). 